\title{
Modèles murins de léiomyomes utérins obtenus par oncogenèse ciblée
}

Le léiomyome ou fibrome utérin est une lésion bénigne de l'utérus rencontrée de manière courante en clinique puisqu'elle est retrouvée chez près de $20 \%$ à $30 \%$ des femmes en âge de procréer [1]. Le fibrome utérin pourrait être dû à une ou plusieurs mutations somatiques touchant une cellule myométriale et perturbant sa prolif ération cellulaire; en effet, l'origine clonale de léiomyomes indépendants a pu être démontrée dans plusicurs cas [1, 2]. De plus, des observations déjà anciennes suggèrent que certains fibromes pourraient avoir un caractère héréditaire. Cependant, l'hétérogénéité des léiomyomes laisse penser que de nombreux locus génétiques pourraient être impliqués dans leur développement. On observe une importante régression des léiomyomes après la ménopause, ce qui indique que les hormones stéroïdes jouent probablement un rôle important dans le contrôle du développement de cette tumeur. L'implication des œestrogènes dans la croissance des fibromes est ainsi suspectée et plusieurs traitements bloquant la production d'œestradiol ont été proposés [1]. Toutefois, le rôle exact des oestrogènes n'est pas encore totalement élucidé. L'hypothèse généralement admise est que l'effet mitotique des œestrogènes est relayé par le contrôle de la production locale de facteurs de croissance. L'absence de modèles animaux appropriés a rendu difficile la compréhension des différents mécanismes de la prolifération cellulaire impliquée dans la formation des léiomyomes.

L'utilisation de l'oncogenèse ciblée est à l'heure actuelle, une technique différents modèles de cancers animaux. Des travaux récents nous ont ainsi permis d'obtenir pour la première fois des souris transgéniques développant systématiquement et très rapidement des léiomyomes [3]. Plusieurs lignées de souris transgéniques exprimant l'antigène $\mathrm{T}$ du virus simien 40 (SV 40) au niveau du myomètre utérin ont été obtenues par micro-injection du gène $T$ mis sous contrôle des séquences régulatrices du gène de la Calbindine $\mathrm{D} 9 \mathrm{~K}$ (CaBP9K). La CaBP9K est une calciprotéine intracellulaire synthétisée en quantité importante dans le duodénum où l'activation de la transcription de son gène est sous le contrôle du métabolite actif de la vitamine D [4]; ce gène est activé également à un niveau plus faible dans l'utérus, principalement dans le myomètre, sous le contrôle de l'œstradiol [5]. L'analyse des séquences régulatrices du gène de la CaBP9K par transgenèse nous a permis de montrer que le promoteur minimum de ce gène permettait de cibler l'expression d'un transgène dans le myomètre utérin [6]. C'est donc ce promoteur minimum que nous avons utilisé pour contrôler l'expression de l'antigène T de SV40 dans le myomètre de plusieurs souris appartenant à des lignées différentes. Ces animaux développent dès l'âge de deux mois des léiomyomes utérins affectant, soit le corps utérin, soit la corne utérine. Des analyses histologiques ont permis de révéler une très forte homologie de ces tumeurs avec celles développées chez la femme. Ces études ont révélé que la structure de cette tumeur est très hétérogène, avec des zones d'intense prolifération juxtaposées à des zones de fibrose riche en matrice extracellulaire. Par immunohistochimie, nous avons pu observer que les zones prolifératives présentent un marquage nucléaire très intense de l'antigène T de SV40 alors que les zones fibreuses sont caractérisées par un marquage nettement moins intense.

Dans ce modèle de souris transgéniques, les léiomyomes présentent un index mitotique élevé qui auraient dû permettre de les classer comme des léiomyosarcomes si on avait utilisé les critères employés en pathologie humaine. Cependant, malgré cet index mitotique témoignant de l'intense prolifération dans cette tumeur, aucune invasion du péritoine ni métastase n'ont été observées parmi plus de cent souris transgéniques analysées. Ces tumeurs ont donc été classées, en toute rigueur, comme des léiomyomes utérins. Rappelons d'ailleurs que le léiomyosarcome utérin est exceptionnel, contrairement à sa forte prévalence parmi les tumeurs musculaires lisses intestinales. Cette différence pourrait témoigner d'une sensibilité spécifique des cellules de la lignée musculaire lisse à la transformation maligne selon leur origine. Par ailleurs, l'expression du transgène est, tout comme le gène de la Calbindine D9K, strictement sous le contrôle des œstrogènes. Un élément de réponse aux œestrogènes, présent dans les séquences régulatrices du gène de la CaBP9K est en effet inclus dans le transgène CaBP9K/SV40 et permet un strict contrôle de son expression. Ainsi l'oncogène T de SV40 est synthétisé, dès la maturité sexuelle, stimulant la croissance des cellules musculaires lisses de l'utérus, alors qu'aucune anomalie n'est observée 


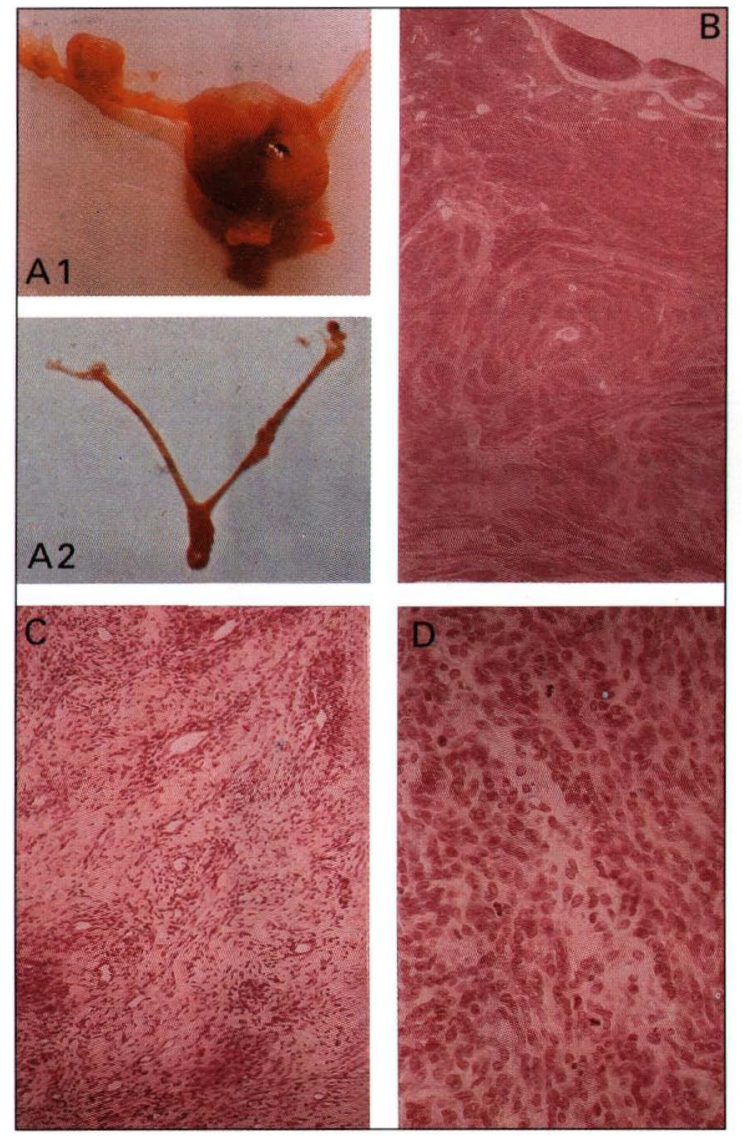

chez des souris transgéniques ovariectomisées ou impubères. En outre, l'ovariectomie d'animaux transgéniques développant des léiomyomes permet de stopper la progression tumorale. Ainsi, l'expression de l'antigène T de SV 40 est non seulement nécessaire au déclenchement mais aussi à la progression du phénotype tumoral. Comme chez la femme, ces léiomyomes dépendent ainsi strictement de la présence des oestrogènes. Chez ces souris transgéniques, les œestrogènes pourraient jouer un double rôle en tant que promoteur tumoral : ils stimuleraient la multiplication cellulaire à la fois par activation de la synthèse de l'antigène $T$ de SV 40 et par un effet indépendant du transgène et spécifique des tissus hormonosensibles, tel le myomètre. De tels effets des oestrogènes semblent relayés par l'induction de la sécrétion de facteurs de croissance et la répression de la production de substances inhibitrices tel le TGF $\beta$ (transforming growth factor $\beta$ ) au niveau de l'épithélium mammaire [7]. Quoique l'inter-
Figure 1. Aspect macroscopique et histologique d'un léiomyome utérin chez des souris transgéniques $\mathrm{Cal}$ bindine $D 9 K / T$ SV 40. A1. Aspect morphologique des léiomyomes. La tumeur se développe majoritairement dans le corps utérin, quelques nodules sont présents dans la corne. A2. Absence de développement de tumeur chez les souris transgéniques ovariectomisées. B. Histologie du léiomyome (coloration hématoxyline-éosine). La tumeur présente une forte hétérogénéité avec des zones $d^{\prime}$ intense prolifération (C. fort agrandissement) juxtaposant des zones de fibrose (D. fort agrandissement).

bien qu'en pathologie tumorale humaine. Ce modèle devrait permettre non seulement une meilleure comprćhension des mécanismes complexes réglant la croissance des fibromes mais aussi de mieux expliquer le mécanisme d'action des agents utilisés aujourd'hui en thérapeutique, les limites de leurs effets et d'entrevoir de nouvelles méthodes thérapeutiques.

B.R. C.P.

1. Rein MS, Nowak RA. Biology of uterine myomas and myometrium in vitro. Semin Reprod lindocrinol $1992 ; 10: 31()-9$

2. Hashimoto K, Azuma C, Kimiura S, Kimura T, Nobumaga T, Kimai T, Sawada M, Noguchi S, SajiF. (Conal determination of uterine leiomyomas by analyzing differential inactivation of the $\mathrm{X}$ chromosome-linked phosphoglycerokinase gene. (innecol (Obstet Invest $1995 ; 40$ : 204-8.

3. Romagnolo B, Molina T, I eroy C, Blin (;, Porteux A, Thomasset M, Vandewalle A, Kahn A, Perret C. Estradiol-dependent uterine leiomyomas in transgenic mice. J Clin Invest 1996; 98: 1-8.

4. Thomasset M, Parkes (.O, Cuisinier-Gleizes P. Rat calcium-binding proteins: distribution, development, and vitamin D) dependence. Am J Physiol. $1982 ; 243:$ F $483-8$

loppente l'antigène $\mathrm{T}$ dans le loppement de ces tumeurs animales ne corresponde évidemment pas à un phénomène rencontré chez les femmes, ce modèle transgénique pourrait néanmoins être plus proche des situations rencontrées en cancérogenèse humaine qu'il n'y paraît. En effet, on sait que l'une des fonctions essentielles de l'antigène $T$ est de former des complexes avec les anti-oncogènes p53 et $\mathrm{Rb}$ [8], inhibant ainsi la fonction anti-oncogénique. Or, le gène $p 53$ est muté dans près de $50 \%$ des léiomyosarcomes humains $[9,10]$. De plus, la protéine Mdm2, dont la fonction principale semble être de se complexer avec p53 et de régler négativement son pouvoir antiprolifératif et pro-apoptotique [8], est produite en quantités anormalement élevées dans certaines tumeurs musculaires lisses humaines dans lesquelles le gène $M d m 2$ est amplifié [10, 11]. Ainsi, l'inhibition de p53 pourrait jouer un rôle essentiel dans le développement tumoral, chez nos souris transgéniques aussi
5. I.'Ilorset F, Blin C, Brehier A, Thomasset M, Perret C. Estrogen-induced ('albindin-D9K gene expression in the rat uterus during the estrous lindocrinology 1993; 132: 489-95.

6. Romagnolo B, (iluzeaud F, I.ambert M, Colnots, Porteux A, Molina T, Thomasset M, V'andewalle A, Kahn A, Perret C. Tissue-specific and hormonal regulation of Calbindin-D9K fusion transgenic mice. I Biol Chem 1996; 271: $16820-(6$

7. May F, Westley BR. Estrogen regulated messenger RNAs in human breast cancer cells. Biomed Pharmacother 1995; 49: 4(0)-14.

8. Soussi T, Lacronique V'. Nouveaux modèles murins d'étude du gène suppresseur de tumeur 153. médecine/sriences $1996 ; 12$ : 215-21.

9. Andreassen A, Oyjord T, Hovig E, Holm R, Florenes V'A, Nesland JM, Myklebost O, Hoic J, Bruland OS, Borresen AI., Fodstad O. p53 abmormalities in different subtypes of human sarcomas. Cancer Res 1993 ; 53 : 468-71.

10. Patterson H, Gill S, Fisher (;, I aw MG, Jayatilake H, Fletcher (.D, Thomas M, Grimer R, Gusterson BA, Cooper C.S. Abnormalities of the $p 53$, $M d m 2$ and $D C C$ genes in human leiomyosarcomas. Br / Cancer $1994 ; 69$ : 1052-8.

11. Leach FS, Tokino T, Meltyer P, Burell M, Oliner JD, Smith S, Hill DE, Sidransky D, Kinzler KW, V'ogelstein B. $p 5.3$ mutations and Mdm2 amplification in human soft tissue sarcomas. Cancer Res $1993 ; 53: 2231-4$ cycle: late antagonistic effect of progesterone. 